\title{
Restless leg syndrome in chronic hemodialysis patients in Mashhad hemodialysis centers
}

\author{
Niloufar Zadeh Saraji ${ }^{1}$, Maryam Hami ${ }^{*}$, Reza Boostani ${ }^{2}$, Mohammad Javad Mojahedi ${ }^{1}$ \\ ${ }^{1}$ Kidney Transplantation Complications Research Center, Mashhad University of Medical Sciences, Mashhad, Iran \\ ${ }^{2}$ Department of Neurology, Ghaem Hospital, Mashhad University of Medical Sciences, Mashhad, Iran
}

\section{AR T I C L E I N F O}

Article Type:

Original

\section{Article History:}

Received: 27 September 2016

Accepted: 8 December 2016

Published online: 25 December 2016

\section{Keywords:}

Restless leg syndrome

Hemodialysis

Chronic kidney disease

Hemodialysis

End-stage renal disease

\begin{abstract}
A B S T RA C T
Introduction: Restless leg syndrome (RLS) is a sensory motor disorder. Patients with this syndrome have serious and uncontrollable desire to move their legs, which is mostly due to an uncomfortable feeling intensified when they are motionless. It may be a genetic disorder or secondary to iron deficiency, neurodegenerations, pregnancy, some drugs and severe kidney diseases.

Objectives: This study was designed to find out the prevalence and its risk factors of RLS in hemodialysis patients.

Patients and Methods: This multicenter cross-sectional study was done on 260 hemodialysis patients. The prevalence of RLS was measured using International Restless Legs Syndrome Study Group (IRLSSG)'s RLS Questionnaire (RLSQ). Potential risk factors for RLS including underlying cause of chronic renal failure, duration on dialysis, biochemical tests, dialysis adequacy, and erythropoietin and also venofer dosage in recent month and demographic data were also evaluated.

Results: The prevalence of RLS was 55\% including 59.4\% males and $40.6 \%$ females. Their mean age of RLS patients and their dialysis duration were significantly higher than other group $(P<0.05)$. Their body mass index $(\mathrm{BMI})$ and serum calcium were significantly higher $(P<0.05)$. However erythropoietin dosage and serum hemoglobin level were lower in RLS patients $(P<0.05)$. Significant predictors of RLS were history of diabetes mellitus (DM), hypertension $(\mathrm{HTN})$, smoking $(P<0.05)$. There was not significant relation between RLS and dialysis adequacy, serum intact parathyroid hormone (iPTH), urea, ferritin and venofer dosage $(P>0.05)$.

Conclusion: According to the results, RLS is a common disorder in hemodialysis patients which can affect strongly on their life. So particular attention and sooner diagnosis of RLS in high risk patients for better management is necessary.
\end{abstract}

Implication for health policy/practice/research/medical education:

In a multicenter cross-sectional study on 260 stable hemodialysis patients to detect the prevalence of RLS and its risk factors, we found its prevalence was $55 \%$ of patients. It is appeared RLS is a common disorder in hemodialysis patients and patients with past history of DM, HTN and smoking are more prone to it. So particular attention and sooner diagnosis of RLS in high risk patients for better management is necessary.

Please cite this paper as: Zadeh Saraji N, Hami M, Boostani R, Mojahedi MJ. Restless leg syndrome in chronic hemodialysis patients in Mashhad hemodialysis centers. J Renal Inj Prev. 2017;6(2):137-141. DOI: 10.15171/jrip.2017.27.

\section{Introduction}

Restless leg syndrome (RLS) is a sensorimotor disturbance with features of both neurologic and sleep disorders. Patients who afflicted describe an intensely uncomfortable urge to move legs predominantly in the evening or at night, which disturbs their sleep. The pattern of movement is involuntary dorsiflexion of foot and lower leg, that lasting
2 to 5 seconds (1-5).

RLS is described in two form of primary and secondary. Primary RLS occurs in patient with positive family history who is usually older than 45 years (2). Secondary RLS can be happened in patients with uremia, pregnancy, iron deficiency state, rheumatoid arthritis, diabetes mellitus (DM) type 2 and some neuropathies (2). In primary RLS, 
dysfunction of the dopaminergic system and reduced iron stores in specific regions in the brain are implicated as pathophysiologic mechanism, however in secondary RLS in uremic patients in spite of dysfunction of dopaminergic system, calcium and phosphate imbalance can help to its initiation and also its severity (6).

RLS is important as it can affect on sleep quality and cardiovascular function, as it can accelerate nocturnal hypertension (HTN), stroke, depression and anxiety in patients (2). It is appeared hemodialysis patients who suffered from RLS, have higher mortality rate than other end-stage renal disease (ESRD) patients (7).

\section{Objectives}

Based on previous studies, prevalence of RLS in general population is between $2.5 \%$ and $15 \%$, which increases with age and probably higher in female gender. Its prevalence is even more in dialysis patients especially when using International Restless Legs Syndrome Study Group (IRLSSG), that are reported about $20 \%$ to $62 \%$ (8). Hence, we tried to find prevalence and related factors in ESRD in hemodialysis centers in Mashhad.

\section{Materials and Methods}

We conducted an observational cross-sectional study on 260 consecutive hemodialysis patients from October to December 2014 at Ghaem, Imam Reza, Montaseriye, Mousabenejafar hospitals, and kidney diseases organization hemodialysis centers in Mashhad, Iran. We enrolled all stable patients were older than 16 year-old with at least 6 months history of chronic hemodialysis in above centers. Patients who were not cooperative, like patients with confusion or dementia were excluded. Also patients taking regular dosage (more than 10 times per month) of anti-histaminic drugs, dopamine agonists, tricyclic antidepressant, benzodiazepines, gabapentin, anti-convulsion and alcohol consumption were excluded. The data collection was carried out by personal interview, using standardized diagnostic questionnaire by IRLSSG. Patients were evaluated by face-to-face interviews. The questionnaire was also consisted of demographic and laboratory data such as age, gender, weight, height, marital status, employment, medication, smoking and coffee or tea intake, the underlying cause of chronic renal failure, duration on dialysis, intravenous iron dosage and erythropoietin dosage in recent month, serum hemoglobin, serum ferritin, serum urea, calcium and phosphate and also dialysis adequacy $(\mathrm{Kt} / \mathrm{V})$.

\section{Ethical issues}

The research followed the tenets of the Declaration of Helsinki. Informed consent was obtained and the research was approved by the Ethics Committee of Mashhad University of Medical Sciences.

\section{Statistical analysis}

Statistical analysis was performed by the SPSS software version 21. Categorical variables were expressed as proportions and compared with the chi-square test. Variables such as age and body mass index (BMI) were expressed as means and standard deviations, and were compared with $t$ tests. $P$ value less than 0.05 were considered statistically significant.

\section{Results}

The total number of patients recruited for the study was 260. The mean age was $48.99 \pm 15.72$ years. There were 157 males $(60.4 \%)$ and 103 females $(39.6 \%)$. The mean duration on dialysis was $47.88 \pm 40.44$ months. The commonest etiology of chronic kidney disease was HTN in $78(30 \%)$ patients and DM in $73(28.1 \%)$ patients. The majority of patients (81.9\%) were married and most of them $(85.8 \%)$ were unemployed. The mean BMI was $22.91 \pm 4.22 \mathrm{~kg} / \mathrm{m}^{2}$. Other demographic and laboratory data are shown in Table 1.

The study results indicated that out of 260 hemodialysis patients 143 (55\%) presented RLS. There were significant relation between causes of kidney disease and RLS, while, patients with history of DM and HTN had significantly higher frequency of RLS $(P=0.001)$. However patients with past history of urologic problems and rheumatologic disease had not this relation $(P>0.05)$. Detail of underlying disease has been compared in patients with and without RLS in Table 2. Totally 72 (27.8\%) of patients were retired. $49(68.1 \%)$ of them had RLS that was significantly higher percentage than other groups $(P=0.035)$ that consist of employed and unemployed patients. In employed group, we did not find significant relation between job and RLS. Married patients had significantly higher symptoms of RLS, as 126 (59.4\%) of 212 married patients had RLS. However in unmarried group $16(34 \%)$ of 47 patients had RLS $(P=0.004)$. While only $12(4.6 \%)$

Table 1. Demographic and laboratory data in patients

\begin{tabular}{lccc}
\hline Characteristics & RLS & No RLS & $\boldsymbol{P}$ \\
\hline $\mathrm{M} / \mathrm{F}$ & $85 / 58$ & $72 / 45$ & 0.731 \\
Age $(\mathrm{y})$ & $51.72 \pm 14.49$ & $45.65 \pm 16.57$ & 0.002 \\
Weight $(\mathrm{kg})$ & $62.94 \pm 13.02$ & $59.91 \pm 12.32$ & 0.057 \\
Height $(\mathrm{cm})$ & $162.42 \pm 16.47$ & $163.90 \pm 9.25$ & 0.386 \\
BMI(kg/m²) & $23.48 \pm 4.34$ & $22.22 \pm 3.99$ & 0.017 \\
Dialysis duration (y) & $4.72 \pm 5.03$ & $3.10 \pm 4.53$ & 0.008 \\
PTH (ng/L) & $485.28 \pm 451.85$ & $476.22 \pm 425.96$ & 0.869 \\
Ferrittin (ng/mL) & $1107 \pm 07$ & $490.80 \pm 428.83$ & 0.093 \\
Hemoglobin (g/ dL) & $11.04 \pm 1.98$ & $11.70 \pm 2.74$ & 0.031 \\
Urea (mg/dL) & $126.78 \pm 34.95$ & $128.72 \pm 37.03$ & 0.665 \\
Calcium(mg/dL) & $8.43 \pm 0.86$ & $8.20 \pm 0.82$ & 0.034 \\
Phosphosrus (mg/ & $5.79 \pm 1.37$ & $5.54 \pm 1.68$ & 0.187 \\
dL) & $1.36 \pm 0.33$ & $1.43 \pm 0.36$ & 0.113 \\
Kt/V & & $10111.11 \pm 6543.98$ & 0.005 \\
EPO dosage (unit/ & $7804.19 \pm 6610.12$ & & \\
wk) & & $555.6 \pm 0.49$ & 0.699 \\
$\begin{array}{l}\text { Venofer dosage (mg/ } \\
\text { mon) }\end{array}$ & $531.5 \pm 0.50$ & & \\
\hline
\end{tabular}


patients were smoker, most of them had RLS (83.3\%) $(P=0.043)$. Patients evaluated for tea consumption, there was not significant relation between frequency of RLS and daily tea consumption $(P=0.165)$. There were no significant differences in prevalence of RLS in patients who took eligible dosage (less than 10 times in month) of antihistaminic drugs, anticonvulsant drugs and tricyclic antidepressant drugs $(P>0.05)$. However, in RLS patients gabapentin consumption was more than other patients $(P=0.02$; Table 3$)$.

\section{Discussion}

This is the first study of prevalence of RLS in hemodialysis patients in Mashhad, north-east of Iran. Previously Zamani et al reported prevalence of RLS in general population in Tehran (Iran) in patients referring to neurology and orthopedic clinics, which was $10.5 \%$ (9). Based on a systematic review in 2010, Innes et al reviewed 34 papers about prevalence of RLS in western countries. They reported prevalence rates ranged from $4 \%$ to $29 \%$ of adults across studies (10).

It is assumed RLS is more common in hemodialysis patients, as uremia is one of the causes of secondary RLS. Chavoshi et al reported the prevalence of RLS was about $31.7 \%$ in hemodialysis patients in Tehran, Iran (2). AlJahdali et al evaluated 227 ESRD patients in Jeddah, Saudi Arabia. The prevalence of RLS was 50.22\% (11). Similar studies in other countries reported prevalence of RLS to be $6 \%$ to $80 \%$ in patients with chronic renal failure (1215), for example $21.5 \%$ in Brazil (16), $14 \%$ in Canada (17), $18.4-21.5 \%$ in Italy (18), 6.6\% in India (19), 20.3\% in Syria (20), $62 \%$ in China (21), and $12 \%-23 \%$ in Japan $(22,23)$. These highly variable results may be due to difference in races, diagnostic criteria of RLS, methods of studies. Our results were in line with Saudi Arabia and China studies. In this study we did not find any association between gender and RLS. However some previous studies $(2,10,11)$ found this relationship. It can be explained by lower proportion of patients.

The mean age of patients with RLS was significantly higher than other group that was similar to previous studies, that RLS were happened more in older dialysis patients $(2,10,11)$. It can be confirmed by higher RLS in retired patients.

Our results revealed that BMI of patients with RLS was significantly higher than patients without RLS. This is compatible with the results of Giannaki et al (6) and Bayard et al (24) that reported RLS was more prevalent in overweight patient. Even it is suggested that weight loss can help to symptom relieve of RLS. Although some other studies did not approve this relationship $(2,11)$.

Based on our results, longer dialysis duration was associated with higher prevalence of RLS. These results are similar to results of Giannaki et al (6) and Gigli et al $(4)$. However other studies $(2,11)$ could not find this association. It can be explained that neuropathy in ESRD patients appears as a late symptom and can be deteriorated
Table 2. Underlying disease and RLS

\begin{tabular}{lll}
\hline & RLS & No RLS \\
\hline HTN & $46(59.1 \%)$ & $31(40.3 \%)$ \\
DM & $26(81.2 \%)$ & $6(18.8 \%)$ \\
Diabetes + HTN & $27(67.5 \%)$ & $13(32.5 \%)$ \\
Urologic diseases & $17(36.2 \%)$ & $30(63.8 \%)$ \\
Trauma & $2(33.3 \%)$ & $4(66.7 \%)$ \\
Other diseases & $23(41.1 \%)$ & $33(58.9 \%)$ \\
\hline
\end{tabular}

Table 3. Drug history and RLS

\begin{tabular}{lccc}
\hline Drug & RLS & No RLS & P value \\
\hline Anti-histaminic drug & $32(22.4 \%)$ & $22(18.8 \%)$ & 0.48 \\
SSRI & $16(11.2 \%)$ & $11(9.4 \%)$ & 0.638 \\
Benzodiazepines & $22(15.4 \%)$ & $11(9.4 \%)$ & 0.149 \\
Anticonvulsant & $9(6.3 \%)$ & $8(6.8 \%)$ & 0.86 \\
TCA & $13(9.1 \%)$ & $5(4.3 \%)$ & 0.128 \\
Gabapentin & $30(21 \%)$ & $10(8.5 \%)$ & 0.006 \\
\hline
\end{tabular}

by hemodialysis as therapeutic method.

We did not find any difference between two groups regarding serum PTH, phosphorus level and RLS syndrome. However difference in serum calcium was significant. Moreover, calcium/phosphate imbalance is also reported to be involved in the pathophysiology of uremic RLS (23). It can be approved by more studies. Results showed there was no correlation between serum urea and dialysis adequacy (Kt/V) and RLS. Previous study showed this association is controversial (6). Hence, it is necessary to conduct larger studies on this aspect of dialysis patients.

Some studies revealed that iron deficiency can lead to RLS, even in the absence of anemia (25). In contrast with Sloand et al, we did not find any association between serum ferritin levels and RLS. However we found significant correlation of serum hemoglobin level and RLS. It can be explained by that serum ferritin level may be affected by other factors such as inflammation, and is not good predictor of iron store (26). Erythropoietin dosage was significantly lower in RLS group. Thus we concluded that, correction of anemia by higher dosage of erythropoietin can decrease or resolve RLS symptoms in dialysis patients. Accordingly, patients with past history of DM had significantly higher prevalence of RLS. Previous studies suggested DM is a dependent risk factor of RLS (11). We found also a significant relation between HTN and RLS that similar results observed before (27).

Chen et al found that smoking was associated with RLS, whereas consumption of coffee and tea had a negative effect on RLS (28). Gigli et al did not find any association between smoking, coffee intake and RLS (4). Al-Jahdali et al found significant correlation between RLS and daily regular use of coffee, but not with cigarette smoking (11). We found significant relation between smoking and RLS, but not tea consumption.

Our RLS patients took anticonvulsant gabapentin 
significantly higher than non-RLS group. It can be explained by effects of Gabapentin on RLS symptoms which relives after it's taking (6). Other drugs that effect on RLS severity were similar in both groups.

\section{Conclusion}

Using the IRLSSG questionnaire to identify dialysis patients with RLS, the prevalence was significantly high. RLS may result poor quality of life. Therefore, physicians taking care of dialysis patients should be aware of RLS to diagnose it as soon as possible. Future research should focus on unfolding the mechanisms underlying the high prevalence of RLS in the uremic patients and better treatment of it.

\section{Limitations of the study}

Some limitations of our study were selection bias from collecting data from only selected dialysis centers; also we did not obtain data on nerve conduction parameters.

\section{Acknowledgments}

This study was the result of the MD thesis of Niloufar Zadeh Saraji.

\section{Authors' contribution}

NZS; acquisition of data and analysis and interpretation of them. $\mathrm{MH}$; conception and design, drafting the article and revising it. RB; conception and design. MJM; final approval of the version.

\section{Conflicts of interest}

The authors declare no conflict of interests.

\section{Ethical considerations}

Ethical issues (including plagiarism, data fabrication, double publication) have been completely observed by the authors.

\section{Funding/Support}

The authors would like to thank the Office of Vice Chancellor for Research of Mashhad University of Medical Sciences for financial support of this study (Grant \# 89148).

\section{References}

1. Winkelman JW. Periodic limb movements in sleep endophenotype for restless legs syndrome? N Engl J Med. 2007;357:703-5. doi: 10.1056/NEJMe078129.

2. Chavoshi F, Einollahi B, Sadeghniat Haghighi K, Saraei M, Izadianmehr N. Prevalence and sleep related disorders of restless leg syndrome in hemodialysis patients. Nephrourol Mon. 2015;7:e24611. doi: 10.5812/numonthly.24611.

3. Unruh ML, Sanders MH, Redline S, Piraino BM, Umans JG, Chami H, et al. Subjective and objective sleep quality in patients on conventional thrice-weekly hemodialysis: comparison with matched controls from the sleep heart health study. Am J Kidney Dis. 2008;52:305-13. doi: 10.1053/j. ajkd.2008.04.019.

4. Gigli GL, Adorati M, Dolso P, Piani A, Valente M, Brotini S, et al. Restless legs syndrome in end-stage renal disease. Sleep Med. 2004;5:309-15.

5. Einollahi B, Izadianmehr N. Restless leg syndrome: a neglected diagnosis. Nephro Urol Mon. 2014;6:e22009. doi: 10.5812/numonthly.22009.

6. Giannaki CD, Hadjigeorgiou GM, Karatzaferi C, Pantzaris MC, Stefanidis I, Sakkas GK. Epidemiology, impact, and treatment options of restless legs syndrome in end-stage renal disease patients: an evidence-based review. Kidney Int. 2014;85:1275-82. doi: 10.1038/ki.2013.394.

7. Walters AS. Co-morbidity, morbidity and mortality in the restlesslegs syndrome. 2nd WASM World Congress; 4-8 February 2007; Bangkok.

8. Earley CJ. Restless leg syndrme. N Engl J Med. 2003;348:21039. doi: 10.1056/NEJMcp021288.

9. Zamani B, Fereshtenezhad SM, Bakhti S, Jamali Danesh Y, Mehrabani M. Determination of the prevalence and associated factors of restless legs syndrome (RLS) in Iranian nontraumatic patients who referred to neurology and orthopedic clinics. Iran J Neurol. 2008;6:91-9.

10. Innes KE, Selfe TK, Agarwal P. Prevalence of restless legs syndrome in North American and Western European populations: a systematic review. Sleep Med. 2011;12:623-34. doi: $\quad 10.1016 /$ j.sleep.2010.12.018.

11. Al-Jahdali HH, Al-Qadhi WA, Khogeer HA, Al-Hejaili FF, Al-Ghamdi SM, Al Sayyari AA. Restless Legs Syndrome in Patients on Dialysis. Saudi J Kidney Dis Transpl 2009;20:378385.

12. Allen RP, Earley CJ. Restless legs syndrome: a review of clinical and pathophysiologic features. J Clin Neurophysiol. 2001;18:128-47.

13. Hui DS, Wong TY, Li TS, Ko FW, Choy DK, Szeto CC, et al. Prevalence of sleep disturbances in Chinese patients with end stage renal failure on maintenance hemodialysis. Med Sci Monit 2002;8:CR331-6.

14. Kavanagh D, Siddiqui S, Geddes CC. Restless legs syndrome in patients on dialysis. Am J Kidney Dis. 2004;43:763-71. doi. org/10.1053/j.ajkd.2004.01.007.

15. Bhowmik D, Bhatia M, Gupta S, Agarwal SK, Tiwari SC, Dash SC. Restless legs syndrome in hemodialysis patients in India: a casecontrolled study. Sleep Med. 2003;4:143-6. doi: 10.1016/ S1389-9457(03)00005-4.

16. Araujo SM, de Bruin VM, Nepomuceno LA, Maximo ML, Daher Ede F, Correia Ferrer DP, et al. Restless legs syndrome in end-stagerenal disease: clinical characteristics and associated comorbidities. Sleep Med. 2010;11:785-90. doi: 10.1016/j. sleep.2010.02.011.

17. Mucsi I, Molnar MZ, Ambrus C, Szeifert L, Kovacs AZ, Zoller $\mathrm{R}$, et al. Restless legs syndrome, insomnia and quality of life inpatients on maintenance dialysis. Nephrol Dial Transplant. 2005;20:571-7. doi: 10.1093/ndt/gfh654.

18. Merlino G, Piani A, Dolso P, Adorati M, Cancelli I, Valente $\mathrm{M}$, et al. Sleep disorders inpatients with end-stage renal disease undergoingdialysis therapy. Nephrol Dial Transplant. 2006;21:184-90. doi: 10.1093/ndt/gfil44.

19. Novak M, Mendelssohn D, Shapiro CM, Mucsi I. Diagnosis and management of sleep apnea syndrome and restless legs syndrome in dialysis patients. Semin Dial. 2006;19:210-6. doi: 10.1111/j.1525-139X.2006.00157.x.

20. Salman SM. Restless legs syndrome in patients on hemodialysis. Saudi J Kidney Dis Transpl. 2011;22:368-72.

21. Hui DS, Wong TY, Ko FW, Li TS, Choy DK, Wong KK, et al. Prevalence of sleep disturbances in chinese patients with 
end-stage renal failure on continuous ambulatory peritoneal dialysis. Am J Kidney Dis. 2000;36:783-8.

22. Kawauchi A, Inoue $Y$, Hashimoto T, Tachibana N, Shirakawa S, Mizutani Y, et al. Restless legs syndrome in hemodialysis patients: health-related quality of life and laboratory dataanalysis. Clin Nephrol. 2006;66:440-6.

23. Takaki J, Nishi T, Nangaku M, Shimoyama $H$, Inada $T$, Matsuyama N, et al. Clinical and psychological aspects of restless legs syndrome in uremic patients on hemodialysis. Am J Kidney Dis. 2003;41:833-9. doi: 10.1016/S02726386(03)00031-3.

24. Bayard M, Avonda T, Wadzinski J. Restless legs syndrome. Am Fam Physician. 2008;78:235-40.

25. Sloand JA, Shelly MA, Feigin A, Bernstein P, Monk RD. A doubleblind, placebo-controlled trial of intravenous iron dextran therapy in patients with ESRD and restless legs syndrome. Am J Kidney Dis. 2004;43:663-70. doi: 10.1053/j. ajkd.2003.11.021.

26. Allen RP, Earley CJ. The role of iron in restless legs syndrome. Mov Disord. 2007;22:S440-8. doi: 10.1002/mds.21607.

27. Batool-Anwar S, Malhotra A, Forman J, Winkelman J, Li Y, Gao X. Restless legs syndrome and hypertension in middleaged women. Hypertension. 2011;58:791-6.

28. Chen WC, Lim PS, Wu WC, Chiu HC, Chen CH, Kuo HY, et al. Sleep behavior disorders in a large cohort of Chinese (Taiwanese) patients maintained by long term hemodialysis. Am J Kidney Dis. 2006;48:277-84. doi: 10.1053/j. ajkd.2006.04.079.

Copyright ( $) 2017$ The Author(s); Published by Nickan Research Institute. This is an open-access article distributed under the terms of the Creative Commons Attribution License (http://creativecommons.org/licenses/by/4.0), which permits unrestricted use, distribution, and reproduction in any medium, provided the original work is properly cited. 